\title{
Targeted overexpression of leukemia inhibitory factor to preserve myocardium in a rat model of postinfarction heart failure
}

\author{
Mark F. Berry, MDa \\ Timothy J. Pirollia \\ Vasant Jayasankar, MD ${ }^{\mathrm{a}}$ \\ Kevin J. Morine ${ }^{\mathrm{a}}$ \\ Mireille A. Moise, MD \\ Omar Fisher, BS ${ }^{a}$ \\ Timothy J. Gardner, MD ${ }^{\mathrm{a}}$ \\ Paul H. Patterson, $\mathrm{PhD}^{\mathrm{b}}$ \\ Y. Joseph Woo, MD
}

Objective: Myocardial infarction leads to cardiomyocyte loss. The cytokine leukemia inhibitory factor regulates the differentiation and growth of embryonic and adult heart tissue. This study examined the effects of gene transfer of leukemia inhibitory factor in infarcted rat hearts.

Methods: Lewis rats underwent ligation of the left anterior descending coronary artery and direct injection of adenovirus encoding leukemia inhibitory factor ( $\mathrm{n}$ $=10)$ or null transgene as control $(\mathrm{n}=10)$ into the myocardium bordering the ischemic area. A sham operation group $(n=10)$ underwent thoracotomy without ligation. After 6 weeks, the following parameters were evaluated: cardiac function with a pressure-volume conductance catheter, left ventricular geometry and architecture by histologic methods; myocardial fibrosis by Masson trichrome staining, apoptosis by terminal deoxynucleotidal transferase-mediated deoxyuridine triphosphate nick-end labeling assay, and cardiomyocyte size by immunofluorescence.

Results: Rats with overexpression of leukemia inhibitory factor had more preserved myocardium and less fibrosis in both the infarct and its border zone. The border zone in leukemia inhibitory factor-treated animals contained fewer apoptotic nuclei $(1.6 \% \pm 0.1 \%$ vs $3.3 \% \pm 0.2 \%, P<.05)$ than that in control animals and demonstrated cardiomyocytes with larger cross-sectional areas $(910$ $\pm 60 \mu \mathrm{m}^{2}$ vs $\left.480 \pm 30 \mathrm{~mm}^{2}, P<.05\right)$. Leukemia inhibitory factor-treated animals had increased left ventricular wall thickness $(2.1 \pm 0.1 \mathrm{~mm}$ vs $1.8 \pm 0.1$ $\mathrm{mm}, P<.05)$ and less dilation of the left ventricular cavity $(237 \pm 22 \mu \mathrm{L}$ vs $301 \pm 16 \mu \mathrm{L}, P<.05)$. They also had improved cardiac function, as measured by maximum change in pressure over time $(3950 \pm 360 \mathrm{~mm} \mathrm{Hg} / \mathrm{s}$ vs $2750 \pm 230$ $\mathrm{mm} \mathrm{Hg} / \mathrm{s}, P<.05)$ and the slopes of the maximum change in pressure over time-end-diastolic volume relationship $(68 \pm 5 \mathrm{~mm} \mathrm{Hg} /[\mathrm{s} \cdot \mu \mathrm{L}]$ vs $46 \pm 6 \mathrm{~mm}$ $\mathrm{Hg} /[\mathrm{s} \cdot \mu \mathrm{L}], P<.05)$ and the preload recruitable stroke work relationship (89 $\pm 10 \mathrm{~mm} \mathrm{Hg}$ vs $44 \pm 4 \mathrm{~mm} \mathrm{Hg}, P<.05)$.

Conclusions: Myocardial gene transfer of leukemia inhibitory factor preserved cardiac tissue, geometry, and function after myocardial infarction in rats. 


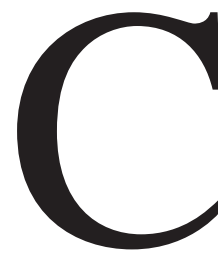

ongestive heart failure (CHF) affects 5 million patients in the United States and eventually occurs in $22 \%$ to $46 \%$ of patients after an acute myocardial infarction (MI). The cardiac dysfunction in ischemic cardiomyopathy is due in part to loss of myocardium at the time of the initial MI. A subsequent remodeling process involving cardiomyocyte apoptosis leads to progressive left ventricular (LV) dilation, fibrosis, and wall thinning, culminating in $\mathrm{CHF}^{2-4}$

The heart has been considered postmitotic and incapable of self-repair, leading to attempts to regenerate functional myocardium by transplanting exogenous cells into injured areas. ${ }^{5,6}$ However, heart transplant tissue has recently been found to contain a significant number of cells that had migrated from the recipient to integrate both as muscle and vascular cells in the transplanted heart. ${ }^{7,8}$ Recent studies have also shown that stem cells both in the heart and mobilized from the bone marrow contribute to myocardial repair. ${ }^{9,10}$ These findings indicate that the heart has some capability for repair, although the signals regulating this process are unknown.

Leukemia inhibitory factor (LIF) is an interleukin 6related cytokine that regulates the differentiation, growth, function, and regeneration of both embryonic and adult tissues. ${ }^{11-13}$ In the heart, LIF induces hypertrophy, blocks apoptosis, and affects the differentiation, proliferation, and maintenance of cardiomyocytes. ${ }^{14-17}$ After acute myocardial injury, LIF downregulates myofibroblast differentiation, collagen deposition, and matrix metalloproteinase activity in cardiac fibroblasts. ${ }^{12}$ LIF also increases expression of vascular endothelial growth factor and manganese superoxide dismutase. ${ }^{18,19}$ Overall, LIF's actions appear to preserve cardiomyocyte integrity and function after injury.

Because of LIF's role in cardiac development, and because LIF's collective effects counteract several processes important in the development of ischemic cardiomyopathy, we hypothesized that LIF may be a key signal in the process of heart regeneration after injury. Indeed, an increase in circulating LIF has recently been shown to induce neovascularization, increase the number of cardiomyocytes in the cell cycle, and enhance the mobilization of bone marrow cells to the heart after MI. ${ }^{20}$ This study was undertaken to determine whether LIF overexpression in the myocardium bordering a large MI would block the subsequent development of heart failure.

\section{Methods}

\section{Animal Care}

This study was conducted according to University of Pennsylvania Institutional Animal Care and Use Committee guidelines. All animals received humane care in compliance with the "Guide for the Care and Use of Laboratory Animals" (http://www.nap.edu/ catalog/5140.html).

\section{Adenoviral Vector Construction}

Recombinant replication-deficient (E1, E3 deleted) adenoviruses containing either the mouse LIF transgene (Adeno.LIF) or the $\beta$-galactosidase reporter gene (Adeno.LacZ), both driven by the cytomegalovirus promoter, were produced in 293 cells and purified as previously described. ${ }^{21}$ Empty replication-deficient adenovirus containing no transgene as a control (Adeno.Null) was obtained from the University of Iowa Gene Transfer Vector core (supported in part by the National Institutes of Health and the Roy J. Carver Foundation).

\section{MI and Adenoviral Delivery}

Thirty-five male Lewis inbred rats (250-300 g, Charles River Laboratories, Inc, Wilmington, Mass) were anesthetized with intraperitoneal injections of ketamine $(50 \mathrm{mg} / \mathrm{kg})$ and xylazine $(5$ $\mathrm{mg} / \mathrm{kg}$ ), intubated, and mechanically ventilated with $0.5 \%$ isoflurane. A left thoracotomy was performed, and the proximal left anterior descending coronary artery was encircled with a 7-0 polypropylene suture. Animals were then randomly assigned in a blinded fashion to one of three experimental groups: control, LIF, or sham. The suture was tied to cause a large anterior MI in the control and LIF groups, after which a total of $5 \times 10^{9}$ plaqueforming units of either Adeno.LIF (LIF group, $n=12$ ) or Adeno.Null (control group, $\mathrm{n}=12$ ) diluted with saline solution to a total volume of $250 \mu \mathrm{L}$ was directly injected into five areas of myocardium bordering the ischemic area. In the sham group $(\mathrm{n}=$ 11), the suture was removed without being tied. All animals had the incisions closed in three layers. Efficacy of viral delivery was confirmed in an additional animal by injection of Adeno.LacZ according to the described method. This rat was killed after 1 week, and X-gal staining on the heart was performed.

\section{Confirmation of In Vivo LIF Transgene Expression With Western Blotting}

LIF viral transgene expression was confirmed in vivo with Western blotting in an additional 6 animals. These animals underwent a left thoracotomy with direct intramyocardial injections of either Adeno.LIF $(n=4)$ or Adeno.Null $(n=2)$ after being anesthetized, intubated, and ventilated as previously described. The animals had their incisions closed and were allowed to recover, and myocardial biopsy specimens were snap frozen in liquid nitrogen after they were killed 1 week later. The frozen biopsy samples were pulverized, homogenized in 10 volumes of sodium dodecylsulfate lysis buffer (100-mmol/L tris[hydroxymethyl]aminomethane, $\mathrm{pH}$ 8.0, $10 \%$ sodium dodecylsulfate, $10-\mathrm{mmol} / \mathrm{L}$ ethylenediaminetetraacetic acid, $50 \mathrm{mmol} / \mathrm{L}$ dithiothreitol), and sheared with a 25 -gauge needle. After specimens were normalized for total protein content, $50 \mu \mathrm{g}$ of each sample were electrophoresed on a $12.5 \%$ sodium dodecylsulfate-polyacrylamide gel after addition of $6 \times$ sample loading buffer and 10 minutes of denaturation at $100^{\circ} \mathrm{C}$. Proteins were transferred to Immobilon-P (Millipore Corporation, Bedford, Mass) with a wet transfer apparatus, and immunoblotting was performed with a goat anti-mouse LIF antibody (R\&D Systems, Minneapolis, Minn). The actin band was identified after staining the Immobilon-P membrane with Coomassie blue to confirm equal loading conditions. 
In Vivo Pressure-Volume Measurements and Analysis

Six weeks after the initial surgery, animals in the two infarct groups and the sham group were again anesthetized, intubated, and mechanically ventilated. A median sternotomy was performed, and a 2F pressure-volume conductance catheter (Millar Instruments, Inc, Houston, Tex) was inserted into the LV through the apex of the heart. Baseline steady-state pressurevolume loops were recorded for each animal. Loops were then recorded while occluding the inferior vena cava (IVC) to reduce preload and allow analysis of load-insensitive measures of ventricular function. IVC occlusion measurements were repeated three to four times for each animal to ensure that pressure-volume loops adequate for subsequent analysis were obtained. Ventilations were held during all recordings to minimize the effects of respiratory variation.

Data was analyzed with ARIA 1 Pressure Volume Analysis software (Millar Instruments). The following parameters were chosen for analysis: maximum LV pressure, ejection fraction, maximum change in pressure over time $(\max \mathrm{dP} / \mathrm{dt})$, and the $\max \mathrm{dP} / \mathrm{dt}$-end-diastolic volume (EDV) relationship as systolic function indices; minimum change in pressure over time (min $\mathrm{dP} / \mathrm{dt}$ ) and the time constant of relaxation $(\tau)$ as diastolic function indices; and preload recruitable stroke work (PRSW) as an index of contractility. PRSW, the slope of the relationship between LV stroke work (SW) and EDV as calculated by linear regression, was chosen to evaluate contractility because it allows measurement of intrinsic ventricular performance independent of loading, geometry, and heart rate and is highly linear in the intact heart. ${ }^{22}$ PRSW was chosen rather than another commonly used contractility measurement, the end-systolic pressure-volume relationship, which is less linear and more dependent on afterload conditions than PRSW and is also highly variable in an intact cardiovascular system, probably because of sympathetic reflexes of the autonomic system. ${ }^{23,24}$ Loops that had the linear regression coefficient for PRSW closest to 1 were chosen for analysis among those recorded.

A cuvette calibration method was used to convert the conductance voltage waveform into volume units. Conductance values for volumes at the low and high ends of the range for rat heart volume were obtained by submerging the conductance catheter into two cylindric nonconductive cuvettes of known diameter filled with heparin-treated rat blood. Subsequent conductance measurements were converted to volumes with a two-point linear interpolation process. Parallel conductance from surrounding structures was calculated by intravenous injection of a small bolus of hypertonic saline solution and subtracted from the measured volume. Volumes measured with this catheter in rat hearts correlate with measurements made with Doppler echocardiography. ${ }^{25}$

After data collection, the heart was arrested in diastole by injection of $0.1 \mathrm{~mL}$ potassium chloride $(1 \mathrm{mEq} / \mathrm{mL})$, and the $\mathrm{LV}$ cavity was filled with Optimal Cutting Temperature embedding compound fixative (Sakura Finetek USA Inc, Torrance, Calif) retrogradely through the transected aortic root. The distended heart was placed in a container of Optimal Cutting Temperature embedding compound fixative and frozen. Sections $10 \mu \mathrm{m}$ thick were prepared with a cryostat through the infarct region from the point of ligation to the apex at $0.25-\mathrm{cm}$ intervals.

\section{Histologic Analysis and Ventricular Wall Thickness Measurements}

Frozen sections from the middle of the infarct region were stained with hematoxylin and eosin and evaluated with light microscopy to assess the histologic effects of LIF overexpression. Sections were also stained with Masson trichrome (Accustain Trichrome Stain [Masson]; Sigma Diagnostics, St Louis, Mo) to distinguish areas of connective tissue. Infarct size in each heart was calculated by averaging the percentages of both inner and outer infarct scar circumferences relative to the LV free wall on two separate sections for each heart with digitized photomicrographs and Openlab image processing software (Improvision, Lexington, Mass). Four measurements of LV free wall thickness in myocardium remote from the infarct were performed on two separate sections as previously described elsewhere. ${ }^{3,4}$

\section{Terminal Deoxynucleotidal Transferase-Mediated Deoxyuridine Triphosphate Nick-End Labeling Assay} The terminal deoxynucleotidal transferase-mediated deoxyuridine triphosphate nick-end labeling (TUNEL) assay was performed on frozen heart sections with a TdT-FragEL DNA Fragmentation Detection Kit (Oncogene Research Products, Boston, Mass) according to the manufacturer's protocol. Counterstaining with methyl green was performed to visualize normal nuclei. The percentages of apoptotic nuclei per total number of nuclei from 10 separate border zone areas on two sections for each heart were averaged.

\section{Cardiomyocyte Size and LIF Immunohistochemical Staining}

Separate frozen sections were fixed with formaldehyde, blocked with $5 \%$ bovine serum albumin in phosphate-buffered saline solution, and incubated either with a mouse antilaminin primary antibody (dilution 1:200 in 5\% bovine serum albumin; NeoMarkers, Fremont, Calif) followed by a rhodamine-conjugated goat antimouse secondary antibody (1:200; Jackson ImmunoResearch Laboratories, West Grove, Pa) or with a goat anti-mouse LIF antibody (1:200; R\&D Systems) followed by a fluorescein-conjugated donkey antigoat secondary antibody (1:200; Jackson ImmunoResearch Laboratories). Sections were mounted with Vectashield mounting medium with 4'-6-diamidino-2-phenylindole (DAPI; Vector Laboratories, Burlingame, Calif), which nonspecifically stains DNA to show cell nuclei. Cardiomyocytes in cross-sectional orientation on lamininstained sections were analyzed with Openlab imaging software (Improvision). Cross-sectional areas of only those cardiomyocytes in which the nucleus was centrally located within the cell were measured. A total of 10 fields from four border zone sections for each heart were analyzed. Approximately 40 cells were measured per heart. The cross-sectional areas of cardiomyocytes in myocardium remote from the infarct scar were also measured in a total of 10 fields on two separate sections for each heart.

\section{Statistical Analysis}

A single, blinded investigator performed all analyses. Funding agencies had no role in data interpretation. All values are expressed as mean \pm SEM. Means of two groups were compared with the unpaired Student $t$ test with a 2-tailed distribution. Com- 
a

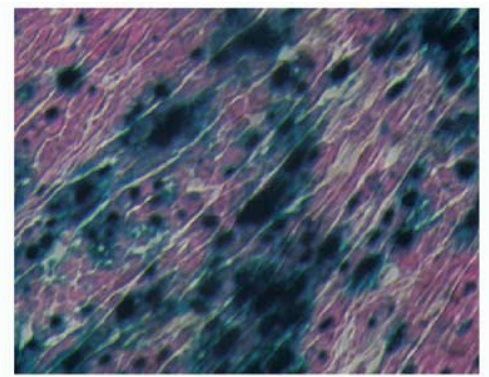

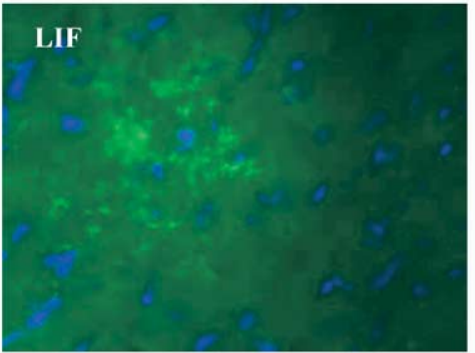

b
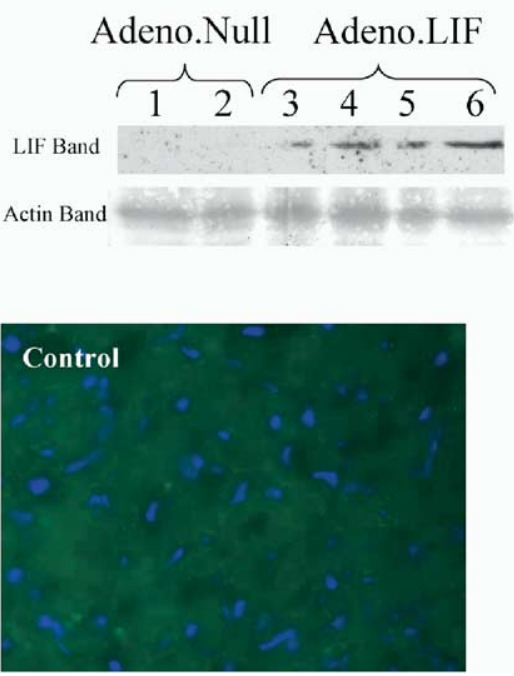

Figure 1. Viral transgene expression after direct intramyocardial injection. a, Rat heart with X-gal staining 1 week after direct injection of Adeno.LacZ. Expression, stained blue, is seen in most cardiomyocytes in LV area of injection. b, Immunoblotting for LIF (43 kd) transgene expression performed 1 week after virus injection shows moderate increase in LIF expression in animals injected with Adeno.LIF (lanes 3, 4, 5, and 6) versus control animals (lanes 1 and 2). Staining of the actin band shows equivalent protein loading in all 6 lanes. c, Merged images of double staining of sections for LIF (green) and nonspecific nuclear stain DAPI (b/ue) demonstrate scattered areas of LIF expression in myocardium bordering infarct in LIF group (left) 6 weeks after MI and viral delivery, whereas control animals (right) do not contain areas of LIF expression (400× magnification).

\section{Control}

a
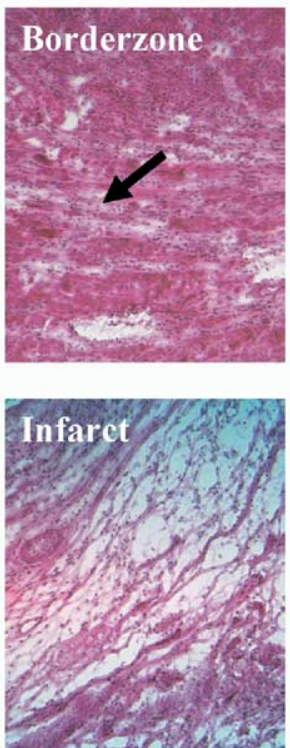

LIF
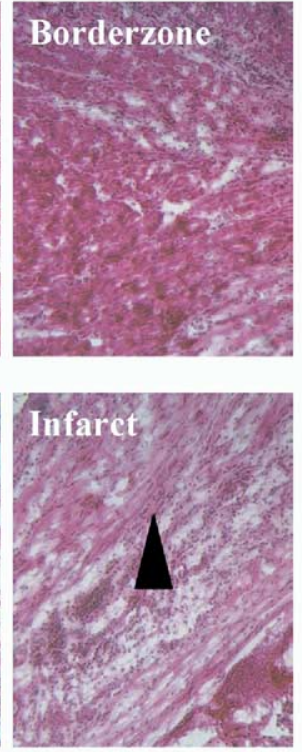

Control

b

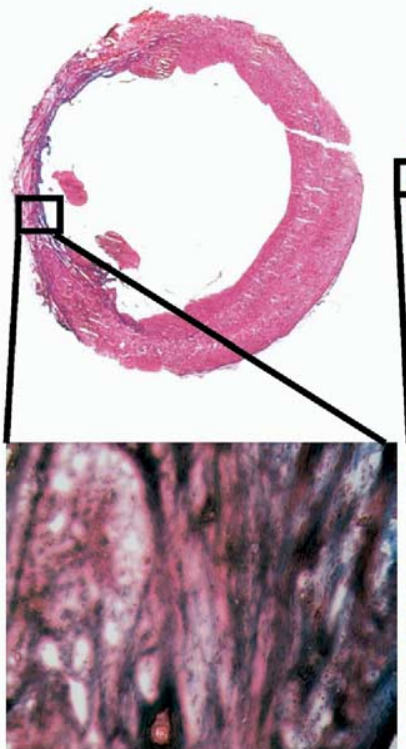

LIF

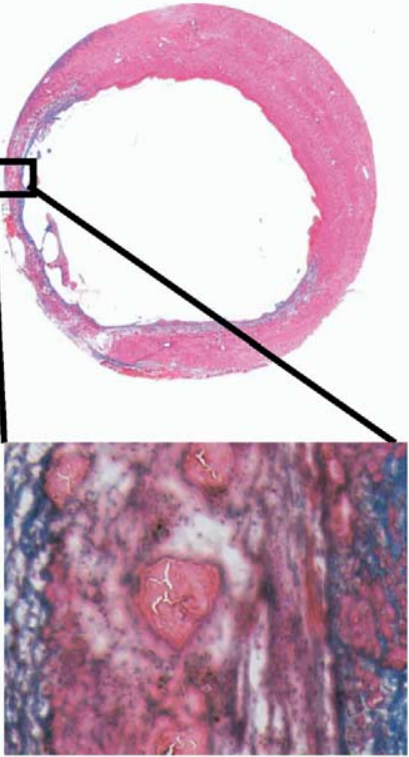

Figure 2. a, Hematoxylin and eosin staining demonstrates more preserved myocardium in both border zone and infarct in LIF group than in control group (100× magnification). Control animals have more connective tissue and fewer cardiomyocytes in border zone than LIF group animals (black arrow). LIF group animals have increased cellularity in infarct relative to control animals (black arrowhead). b, Representative cross-sections with Masson trichrome staining (100 $\times$ magnification) of single rat heart 6 weeks after initial surgery from control group and from LIF group show qualitatively less fibrosis with LIF. Fibrotic areas are stained blue. 
TABLE 1. Hemodynamics and contractility of rats injected with LIF versus sham and control groups

\begin{tabular}{lccc}
\hline & Sham $(\mathbf{n}=\mathbf{1 0})$ & Control $(\mathbf{n}=\mathbf{1 0})$ & LIF $(\mathbf{n}=\mathbf{1 0})$ \\
\hline Heart rate (beats/min) & $196 \pm 9$ & $204 \pm 5$ & $202 \pm 17$ \\
Maximum pressure (mm Hg) & $91 \pm 4$ & $74 \pm 4^{*}$ & $85 \pm 3$ \\
EDV $(\mu \mathrm{L})$ & $200 \pm 5$ & $301 \pm 16^{*}$ & $19 \pm 2^{*}$ \\
Ejection fraction (\%) & $29 \pm 3$ & $2750 \pm 230^{*}$ & $30 \pm 5$ \\
Max dP/dt (mm Hg/s) & $4530 \pm 360$ & $-2290 \pm 240^{*}$ & $3950 \pm 360^{*} \dagger$ \\
Min dP/dt (mm Hg/s) & $-4650 \pm 400$ & $20.4 \pm 1.5^{*}$ & $-3400 \pm 350^{*}$ \\
$\tau(\mathrm{ms})$ & $15.8 \pm 1.0$ & $44 \pm 4^{*}$ & $17.2 \pm 1.1$ \\
PRSW (mm Hg) & $64 \pm 15$ & $260 \pm 11^{*}$ & $89 \pm 10 \dagger$ \\
PRSW volume intercept ( $\mu \mathrm{L})$ & $88 \pm 17$ & $46 \pm 6^{*}$ & $181 \pm 26^{*} \dagger$ \\
Slope max dP/dt-EDV (mm Hg/[s $\cdot \mu \mathrm{L}])$ & $61 \pm 4$ & $216 \pm 10^{*}$ & $68 \pm 5 \dagger$ \\
Max dP/dt-EDV volume intercept $(\mu \mathrm{L})$ & $112 \pm 10$ & & $155 \pm 18 \dagger$ \\
\hline
\end{tabular}

All values are mean \pm SEM.

${ }^{*} P<.05$ versus sham.

$\dagger P<.05$ versus control.

parisons between more than two groups were analyzed by analysis of variance followed by Tukey-Kramer post hoc testing. All statistical analyses were performed with GraphPad InStat (GraphPad Software, Inc, San Diego, Calif).

\section{Results}

\section{Animal Mortality}

Five of the 35 animals in the main study group died in the immediate postoperative period; 2 had received Adeno.LIF, 2 had received Adeno.Null, and 1 had undergone sham operation. Animals that died had their chests immediately reopened, and none had an obvious cause of death. Deaths were presumed to be from a malignant arrhythmia or acute respiratory insufficiency related to the thoracotomy and heart manipulation. All animals that survived the perioperative period lived until the time of hemodynamic measurements. Hemodynamics could not be measured in 1 animal in the LIF group because of significant bleeding after sternotomy during the second operation. This animal's heart, however, was procured and used for tissue analysis.

\section{Adenoviral Delivery and Transgene Expression}

$\mathrm{X}$-gal staining of the animal that received Adeno.LacZ confirmed that the viral delivery method resulted in significant cardiomyocyte transgene transfection (Figure 1,a). Western blotting for LIF transgene expression levels in LV myocardial biopsy samples 1 week after injection showed increased levels of LIF expression in the LIF relative to the control group (Figure 1,b). LIF immunofluorescent staining 6 weeks after MI and viral delivery, a time when adenoviral transgene expression is probably significantly less than maximum, showed that LIF group animals had scattered areas of LIF expression in the myocardium bordering the infarct (Figure 1,c).

\section{Effect on Myocardium}

Infarct size was not significantly different between the two MI groups (LIF $42 \% \pm 2 \%$ vs control $40 \% \pm 3 \%$ of LV free wall). Histologic analysis showed more preserved myocardium in both the infarct and border zone in the LIF group than in the control group (Figure 2,a). Masson trichrome staining qualitatively revealed reduced collagen deposition in the infarct in the LIF group (Figure 2,b). Animals in the LIF group had significantly fewer apoptotic nuclei in the border zone myocardium $(1.6 \% \pm 0.1 \%$ vs $3.3 \% \pm 0.2 \%, P<.05$; Figure 3$)$. Cardiomyocytes in the border zone myocardium of the LIF group animals had significantly greater cross-sectional areas than border zone cardiomyocytes of the control animals (910 $\pm 60 \mu \mathrm{m}^{2}$ vs $480 \pm 30 \mu \mathrm{m}^{2}, P<.001$; Figure 4). LIF group border zone cardiomyocytes also had increased cross-sectional areas relative to cardiomyocytes in myocardium remote from the infarct in both LIF group animals $\left(910 \pm 60 \mu \mathrm{m}^{2}\right.$ vs 460 $\left.\pm 10 \mu \mathrm{m}^{2}, P<.001\right)$ and control animals $\left(910 \pm 60 \mu \mathrm{m}^{2}\right.$ vs $\left.430 \pm 30 \mu \mathrm{m}^{2}, P<.001\right)$.

\section{Cardiac Geometry and Function}

Control animals had significantly decreased cardiac function relative to the sham group (Table 1). Compared with control animals, those in the LIF group had significantly preserved cardiac contractility and hemodynamics (Table 1 and Figures 5 and 6). Pressure-volume loops obtained during IVC occlusion were shifted downward (decrease in LV end-systolic pressure) and rightward (increase in LV EDV) for the control group compared with the sham group, whereas the LIF group had much less downward and rightward shifting than the control group (Figure 5, a). Animals in the LIF group had significantly preserved systolic function, as measured by max $\mathrm{dP} / \mathrm{dt}$ (Table 1 and Figure 5,b) and the max dP/dt-EDV relationship, which was determined from pressure-volume loops obtained 


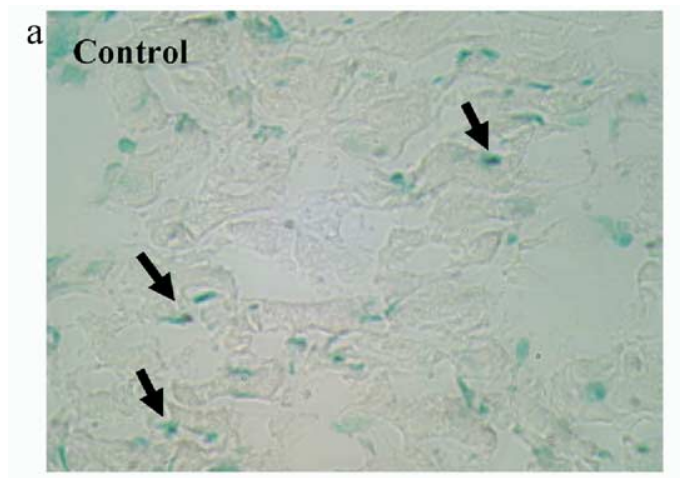

C
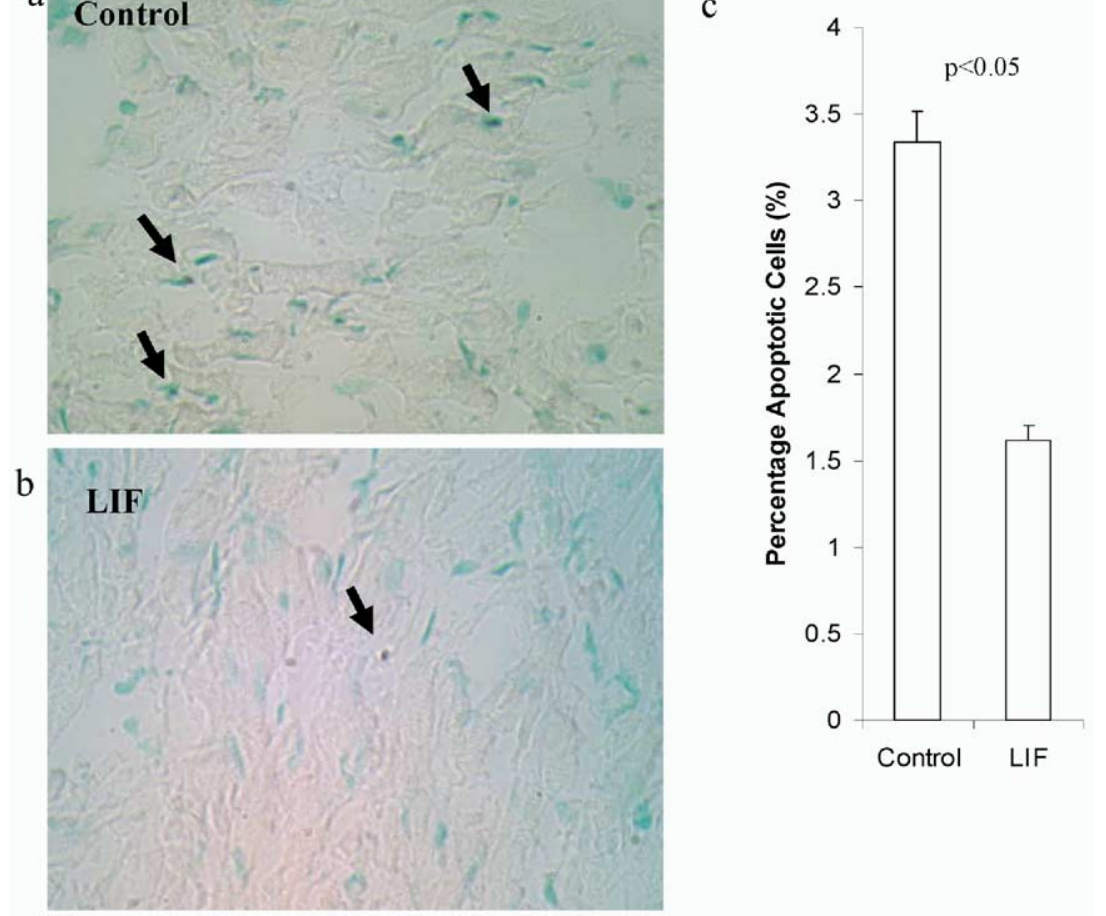

Figure 3. Representative TUNEL-stained cross-sections from single animal in control group (a) and LIF group (b) (400 $\times$ magnification). Apoptotic nuclei are stained dark brown (arrows), and normal nuclei are stained blue-green. c, LIF group animals had smaller percentage of TUNEL-positive cells in infarct border zone area than control animals.
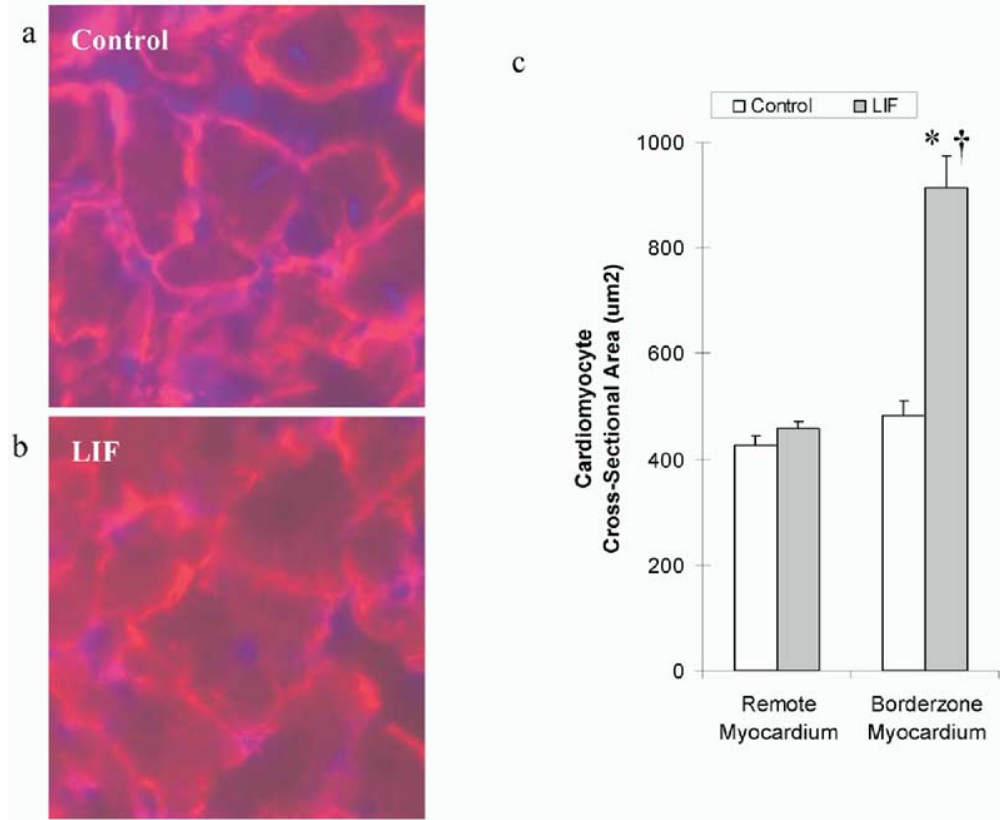

Figure 4. Merged images of double staining of sections for laminin (red) and nonspecific nuclear stain DAPI (blue) demonstrate significantly smaller cardiomyocytes in border zone of control group (a) than LIF group (b) (400X magnification). c, Border zone cardiomyocytes in LIF group animals had higher average cross-sectional areas than border zone cardiomyocytes in control animals, as well as cardiomyocytes in myocardium remote from the infarct in both LIF and control groups. ${ }^{*} \boldsymbol{P}<.001$ versus control border zone; $\boldsymbol{t} \boldsymbol{P}<.001$ versus LIF remote. 

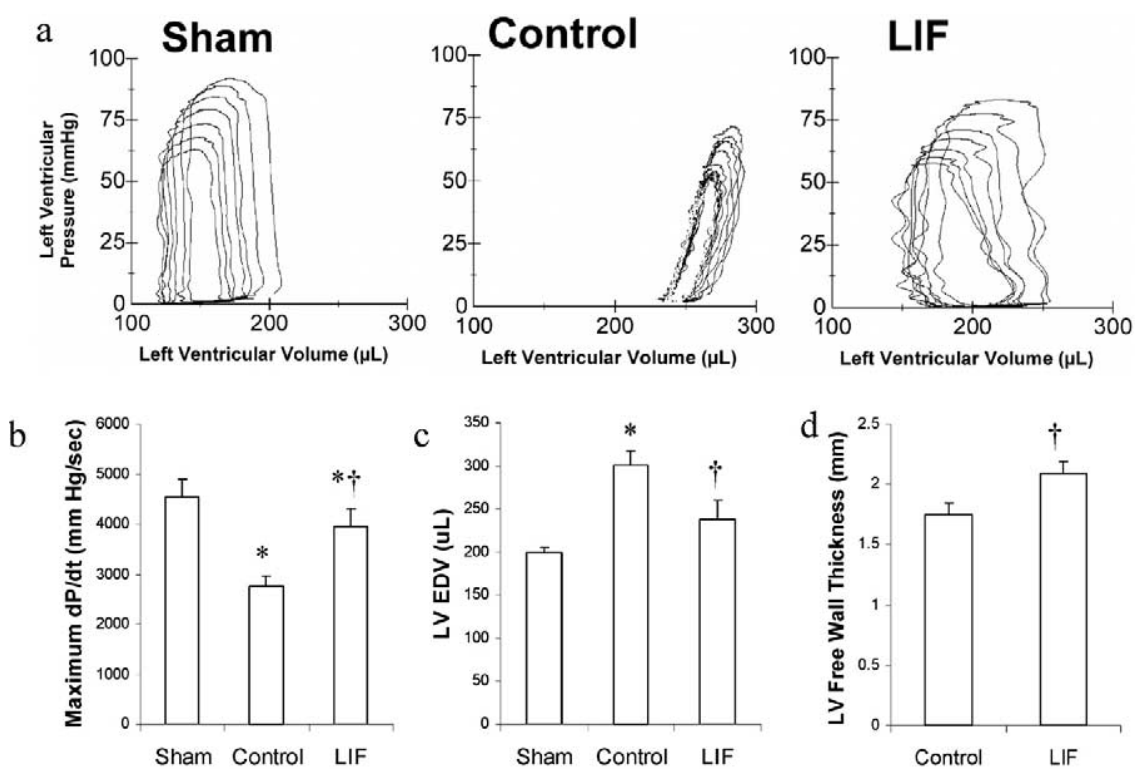

Figure 5. a, Representative pressure-volume loops for sham, control, and LIF groups. Loops for LIF are shifted less than control relative to sham. $\mathrm{b}$, LIF group had improved systolic function, as measured by max $\mathrm{dP} / \mathrm{dt}$, relative to control group. c, LIF group had less ventricular dilation, as measured by LV EDV, than control group. d, LIF attenuated amount of LV wall thinning in myocardium remote from infarct relative to control group. ${ }^{*} \boldsymbol{P}<.05$ versus sham; $\uparrow P<.05$ versus control.

during preload reduction through IVC occlusion. For this relationship, the LIF group had an increased slope and a decreased volume intercept relative to the control group, resulting in less rightward shift (Figure 6, $a$ and $b$ ). Analysis of the SW-EDV relationship, which was also determined from pressure-volume loops obtained during IVC occlusion, demonstrated that the LIF group had improved cardiac contractility (Figure 6, $c$ and $d$ ). The LIF group had an increase in the slope of this relationship, which is PRSW, as well as a decreased volume intercept compared with controls, again resulting in less rightward shift. For the diastolic indices of function min $\mathrm{dP} / \mathrm{dt}$ and $\tau$, the LIF group animals had a trend toward improvement relative to control animals (Table 1); however, this difference did not reach statistical significance.

LIF group animals also had greater preservation of LV geometry than control animals. LIF reduced the amount of $\mathrm{LV}$ dilation after MI, as assessed by in vivo measurements of LV EDV from the conductance catheter (Table 1 , Figure $5, c$ ). LIF also attenuated the amount of LV free wall thinning in myocardium remote from the infarct $(2.1$ \pm 0.1 vs $1.8 \pm 0.1 \mathrm{~mm}, P<.05$, Figure $5, d$ ).

\section{Discussion}

CHF occurs most commonly as a result of post-MI remodeling. ${ }^{1-4}$ Recent studies suggest that the heart has some innate repair mechanism, although the signaling mechanism is unknown. ${ }^{9,10}$ LIF is a cytokine that regulates the differentiation and growth of cardiac tissue and may also regulate cardiac repair. ${ }^{12-20}$ In this study, myocardial overexpression of LIF in a rat model of ischemic cardiomyopathy provided multiple benefits. At the cellular level, LIF overexpression resulted in less infarct fibrosis, as well as cardiomyocyte hypertrophy and less cell loss from apoptosis in the myocardium bordering the infarct. This preservation of heart muscle resulted in less ventricular wall thinning and chamber dilation after MI, as well as preservation of cardiac systolic function and contractility as measured in vivo by multiple parameters with a pressure-volume conductance catheter. Overall, LIF attenuated the development of heart failure after MI.

Interestingly, LIF expression in the heart is increased in human patients with heart failure. ${ }^{26}$ LIF signaling is through glycoprotein 130 receptor activation of two signal transduction intermediate families: the Janus kinase and signal transducers and the activators of transcription and mitogenactivated protein kinase groups. ${ }^{16,27}$ LIF's effects oppose those of mechanical stretch and angiotensin II, which are important in the development of heart failure. The hypertrophy and antiapoptotic phenotypes induced by LIF are blocked by suppressor of cytokine signaling 3 , a mechanical stress-inducible cardiomyocyte gene that inhibits Janus ki- 
a

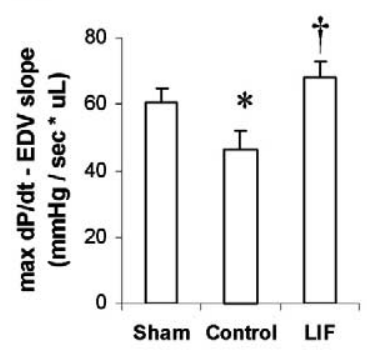

c

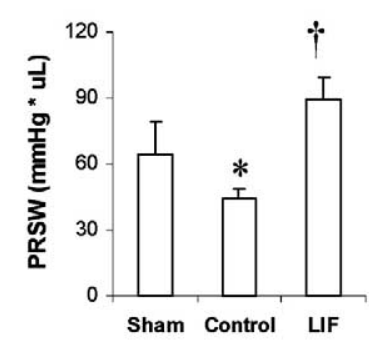

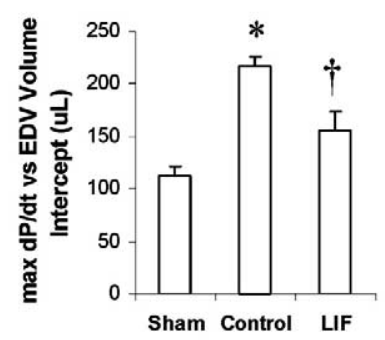

b

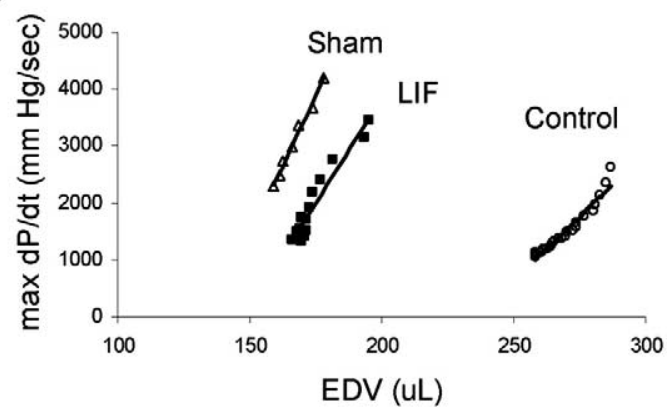

d

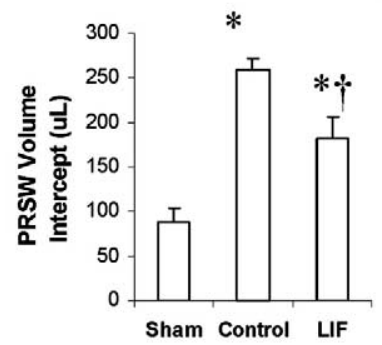

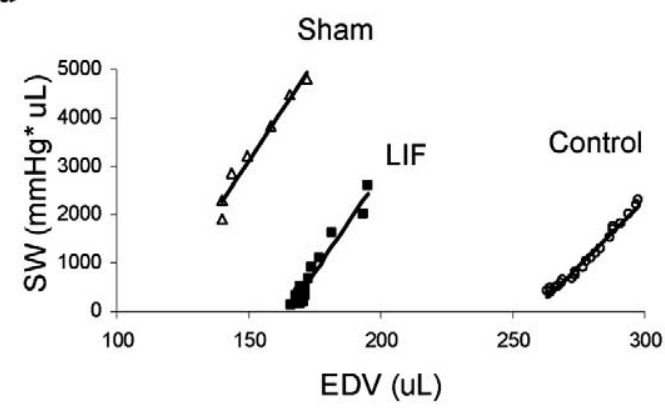

Figure 6. a, LIF group shows increased slope and decreased volume intercept for max dP/dt and EDV relationship relative to control group, indicating improved systolic function. $b$, Representative max dP/dt-EDV relationships for single animal in each experimental group show LIF group shifted rightward and downward less than control group relative to sham group. Each line on graph represents data points for max dP/dt and EDV measured from individual pressure-volume loops obtained during reduction of preload volume by IVC occlusion for single animal from each experimental group. c, LIF group shows increase in slope of SW-EDV relationship, which is PRSW, as well as decreased volume intercept relative to control group, indicating increased contractility. d, Representative SW-EDV relationships for single animal in each experimental group show LIF group shifted rightward and downward less than control group relative to sham group. Each line on graph represents data points for SW and EDV measured from individual pressure-volume loops obtained during reduction of preload volume by IVC occlusion for single animal from each experimental group. ${ }^{*} P<.05$ versus sham; $\uparrow P<.05$ versus control.

nase. ${ }^{28}$ Angiotensin II inhibits LIF-mediated cytoprotective effects. ${ }^{29}$ Although LIF is already upregulated in some forms of CHF, this study suggests that increased expression in the myocardium bordering the infarct may be more helpful, especially considering that the increased mechanical stretch and angiotensin II levels that accompany heart failure probably inhibit LIF's beneficial effects. Targeting LIF expression to the border zone may also prevent any diastolic dysfunction that could result from inducing hypertrophy and blocking apoptosis throughout the heart.

This study does have limitations. First, the source of preserved myocardium was not directly investigated and could have been from bone marrow cell recruitment, proliferation of existing cardiomyocytes, or blocked apoptosis of border zone cardiomyocytes. Although in a recent study LIF appeared to enhance mobilization of bone marrow cells to the heart, another recent study showed that blocking the apoptosis of transplanted bone marrow cells significantly enhanced their effect of regenerating myocardium. ${ }^{6,20}$ LIF may not increase cardiac recruitment of bone marrow cells, but rather may prevent their death once they reach the heart. This issue should be evaluated in a future study. Another limitation is that adenovirus use allowed effective shortterm gene transfer, but significant expression is unlikely to persist beyond 4 weeks. Whether the effects of short-term LIF overexpression are transient or permanent was not determined. Longer term studies are needed to see whether short-term overexpression permanently blocks or simply delays the onset of heart failure. Finally, virally mediated gene transfer may not provide optimal clinical applicability. LIF may have some potential for ultimate systemic use because it works on cell membrane receptors. This study's results may more importantly help further knowledge into the molecular mechanisms of the postinfarction remodeling process.

MI leads to myocardial necrosis and apoptosis and ultimately to heart failure. LIF is a cytokine that regulates heart 
tissue by inducing cardiomyocyte proliferation and hypertrophy, blocking apoptosis, modulating cardiac fibroblast activity, and mobilizing bone marrow cells to the heart. Our results show that LIF gene transfer preserves both myocardium and cardiac function after MI.

We are grateful to Dr. Bradley Kerr for his assistance in providing the LIF adenovirus. We also acknowledge the McGrath Foundation's support of Dr Patterson's laboratory.

\section{References}

1. American Heart Association. Heart disease and stroke statistics-2004 update. Dallas: The Association; 2003.

2. St John Sutton M, Pfeffer MA, Moye L, Plappert T, Rouleau JL, Lamas G, et al. Cardiovascular death and left ventricular remodeling two years after myocardial infarction: baseline predictors and impact of long-term use of captopril: information from the Survival and Ventricular Enlargement (SAVE) trial. Circulation. 1997;96:3294-9.

3. Chatterjee S, Bish LT, Jayasankar V, Stewart AS, Woo YJ, Crow MT, et al. Blocking the development of postischemic cardiomyopathy with viral gene transfer of the apoptosis repressor with caspase recruitment domain. J Thorac Cardiovasc Surg. 2003;125:1461-9.

4. Jayasankar V, Woo YJ, Bish LT, Pirolli TJ, Chatterjee S, Berry MF, et al. Gene transfer of hepatocyte growth factor attenuates postinfarction heart failure. Circulation. 2003;108(Suppl 1):II230-6.

5. Taylor DA, Atkins BZ, Hungspreugs P, Jones TR, Reedy MC, Hutcheson KA, et al. Regenerating functional myocardium: improved performance after skeletal myoblast transplantation. Nat Med. 1998; 4:929-33.

6. Mangi AA, Noiseux N, Kong D, He H, Rezvani M, Ingwall JS, et al. Mesenchymal stem cells modified with Akt prevent remodeling and restore performance of infarcted hearts. Nat Med. 2003;9:1195-201.

7. Muller P, Pfeiffer P, Koglin J, Schafers HJ, Seeland U, Janzen I, et al. Cardiomyocytes of noncardiac origin in myocardial biopsies of human transplanted hearts. Circulation. 2002;106:31-5.

8. Quaini F, Urbanek K, Beltrami AP, Finato N, Beltrami CA, NadalGinard B, et al. Chimerism of the transplanted heart. $N$ Engl J Med. 2002;346:5-15.

9. Orlic D, Kajstura J, Chimenti S, Limana F, Jakoniuk I, Quaini F, et al. Mobilized bone marrow cells repair the infarcted heart, improving function and survival. Proc Natl Acad Sci U S A. 2001;98:10344-9.

10. Beltrami AP, Barlucchi L, Torella D, Baker M, Limana F, Chimenti S, et al. Adult cardiac stem cells are multipotent and support myocardial regeneration. Cell. 2003; 114:763-76.

11. Yamamori T, Fukada K, Aebersold R, Korsching S, Fann MJ, Patterson $\mathrm{PH}$. The cholinergic neuronal differentiation factor from heart cells is identical to leukemia inhibitory factor. Science. 1989;246:1412-6.

12. Wang F, Trial J, Diwan A, Gao F, Birdsall H, Entman M, et al. Regulation of cardiac fibroblast cellular function by leukemia inhibitory factor. J Mol Cell Cardiol. 2002;34:1309-16.

13. Gadient RA, Patterson PH. Leukemia inhibitory factor, interleukin-6, and other cytokines using the GP130 transducing receptor: roles in inflammation and injury. Stem Cells. 1999;17:127-37.

14. Bader A, Al-Dubai H, Weitzer G. Leukemia inhibitory factor modulates cardiogenesis in embryoid bodies in opposite fashions. Circ Res. 2000;86:787-94.

15. Wang F, Seta Y, Baumgarten G, Engel DJ, Sivasubramanian N, Mann DL. Functional significance of hemodynamic overload-induced expression of leukemia-inhibitory factor in the adult mammalian heart. Circulation. 2001;103:1296-302.

16. Kodama H, Fukuda K, Pan J, Makino S, Baba A, Hori S, et al. Leukemia inhibitory factor, a potent cardiac hypertrophic cytokine, activates the JAK/STAT pathway in rat cardiomyocytes. Circ Res. 1997;81:656-63.

17. Negoro S, Oh H, Tone E, Kunisada K, Fujio Y, Walsh K, et al. Glycoprotein 130 regulates cardiac myocyte survival in doxorubicininduced apoptosis through phosphatidylinositol 3-kinase/Akt phos- phorylation and Bcl-xL/caspase-3 interaction. Circulation. 2001;103:555-61.

18. Negoro S, Kunisada K, Fujio Y, Funamoto M, Darville MI, Eizirik DL, et al. Activation of signal transducer and activator of transcription 3 protects cardiomyocytes from hypoxia/reoxygenation-induced oxidative stress through the upregulation of manganese superoxide dismutase. Circulation. 2001;104:979-81.

19. Funamoto M, Fujio Y, Kunisada K, Negoro S, Tone E, Osugi T, et al. Signal transducer and activator of transcription 3 is required for glycoprotein 130-mediated induction of vascular endothelial growth factor in cardiac myocytes. J Biol Chem. 2000;275:10561-6.

20. Zou Y, Takano H, Mizukami M, Akazawa H, Qin Y, Toko H, et al. Leukemia inhibitory factor enhances survival of cardiomyocytes and induces regeneration of myocardium after myocardial infarction. Circulation 2003;108:748-53.

21. Zhu M, Oishi K, Lee SC, Patterson PH. Studies using leukemia inhibitory factor (LIF) knockout mice and a LIF adenoviral vector demonstrate a key anti-inflammatory role for this cytokine in cutaneous inflammation. J Immunol. 2001;166:2049-54.

22. Glower DD, Spratt JA, Snow ND, Kabas JS, Davis JW, Olsen CO, et al. Linearity of the Frank-Starling relationship in the intact heart: the concept of preload recruitable stroke work. Circulation. 1985;71:9941009.

23. Takeuchi M, Odake M, Takaoka H, Hayashi Y, Yokoyama M. Comparison between preload recruitable stroke work and the end-systolic pressure-volume relationship in man. Eur Heart J. 1992;13 Suppl E:80-4.

24. Spratt JA, Tyson GS, Glower DD, Davis JW, Muhlbaier LH, Olsen $\mathrm{CO}$, et al. The end-systolic pressure-volume relationship in conscious dogs. Circulation. 1987;75:1295-309.

25. Cingolani OH, Yang XP, Cavasin MA, Carretero OA. Increased systolic performance with diastolic dysfunction in adult spontaneously hypertensive rats. Hypertension. 2003;41:249-54.

26. Eiken HG, Oie E, Damas JK, Yndestad A, Bjerkeli V, Aass H, et al. Myocardial gene expression of leukaemia inhibitory factor, interleukin-6 and glycoprotein 130 in end-stage human heart failure. Eur J Clin Invest. 2001;31:389-97.

27. Kunisada K, Hirota H, Fujio Y, Matsui H, Tani Y, Yamauchi-Takihara $\mathrm{K}$, et al. Activation of JAK-STAT and MAP kinases by leukemia inhibitory factor through gp130 in cardiac myocytes. Circulation. 1996;94:2626-32.

28. Yasukawa H, Hoshijima M, Gu Y, Nakamura T, Pradervand S, Hanada $\mathrm{T}$, et al. Suppressor of cytokine signaling-3 is a biomechanical stress-inducible gene that suppresses gp130-mediated cardiac myocyte hypertrophy and survival pathways. J Clin Invest. 2001;108:1459-67.

29. Tone E, Kunisada K, Fujio Y, Matsui H, Negoro S, Oh H, et al. Angiotensin II interferes with leukemia inhibitory factor-induced STAT3 activation in cardiac myocytes. Biochem Biophys Res Commun. 1998;253:147-50.

\section{Discussion}

Dr Mark Ratcliffe (San Francisco, Calif). Do you have any idea what the blood flow in the border zone is doing in these animals? One of the really interesting things that came out of Gorman's work at Penn was in sheep where the border zone was seen to be normally perfused. The sheep doesn't have any preformed intraarterial collaterals, and I am not sure whether the rat does. But that border zone went on to remodel, presumably because of the phenomenon that was discussed in an earlier presentation, specifically abnormal strain and stress patterns. Do you think that is what is happening here? Do you have any information about blood flow?

Dr Berry. We did not measure any blood flow parameter in this study, either by counting capillaries or using microcrystals to quantify blood flow. We have found in other studies with this model that the use of angiogenic agents resulted in an improve- 
ment in function, suggesting that increased vascularization of the border zone will alter the remodeling process.

Dr Ross M. Ungerleider (Portland, Ore). I am curious about one thing related to your model, and this is probably because I haven't worked with a rat model, but if you ligate the left anterior descending coronary artery and the animals survive, then how appropriate a model is that? I would imagine that that would be a fairly serious ischemic injury. So maybe there is a lot of collateralization or something that allows these animals to survive, and I am just wondering how applicable that is to more human situations.

Dr Berry. Where the artery is ligated does determine the survival of the animals. We have found that ligating too high on the left anterior descending coronary artery, around the level of the pulmonary artery, results in a mortality as high as $50 \%$. We try to ligate in the same point in the middle of the left anterior descending coronary artery in all animals, and we now have about a $15 \%$ mortality overall, which we find to be acceptable in terms of both our animals and our experimental reagents.

In terms of clinical applicability, we were most interested in studying LIF's effects as the ventricle changes by comparing them to what happens if you don't do anything to the border zone area. In this study, adenovirus, which we don't really see much clinical utility for in the long term, was used for proof of principle.

One of the things that excites us about LIF is that it is an extracellular protein that doesn't need to get inside the cell to exert its effects. LIF as a pharmacologic agent therefore might be able to ultimately provide benefit, at least if it does prove to have benefit in larger animals and eventually in humans.

Dr Robert C. Robbins (Stanford, Calif). You injected this right at the time you created the infarct. Clinically, we are really not going to be there at the time. Potentially you could do this in the catheter lab, and that might have some utility to preserve myocardial function, but surgically long term, have you done any studies where you ligated the LAD and then came back, say, a month later?

Dr Berry. No, we haven't performed those types of studies. However, one advantage in this case of using adenovirus is that you don't really start getting expression for a couple of days. So even though we delivered the vector at the time of the MI, we would expect the treatment really to start a few days later and probably only last for a couple of weeks.

Dr Michael Mann (San Francisco, Calif). I have two somewhat mechanism-related questions. First, the control animals that had ischemia all apparently were treated with a control adenovirus, which itself can cause inflammation and may have a deleterious effect downstream on ventricular recovery. I am wondering whether you ever compared your treated hearts with vehicle or non-adenovirus infected hearts?

Second, LIF, as you know, acts through upregulated expression of a number of target genes. Did you look for upregulated expression of any of those key targets in the hearts that you did treat?

Dr Berry. To answer the first question, we didn't do any experiments in which we just injected vehicle; we used only the Adeno.Null virus as a control. We have done studies not involving adenovirus with this model, though, in which the control animals have just received injections of saline or phosphate-buffered saline solution, and we find that the function in those control groups is pretty much equivalent to the function that we see in the animals that get the null virus. We did have concerns initially that the Adeno.Null virus might actually be causing negative effects that would cause us to overestimate the effects of our treatment, but now we don't think that that is the case in our model.

We haven't looked at other intracellular markers, and that is something that we want to follow up on in response to these results.

Dr Robbins. I have a technical question about your assessment of apoptosis. I am assuming it was consistently done in the border zone for both groups, but can you comment on the use of just TUNEL to differentiate between apoptosis and necrosis, which has been a big criticism? We have used in situ oligo ligation TUNEL and other caspase analysis of apoptosis, and you just use TUNEL.

Dr Berry. We have found previously in our lab with some other techniques that the TUNEL assay gave pretty equivalent measurements of apoptosis. We also have heard that criticism of TUNEL, and we are trying to develop some caspase assays to corroborate the TUNEL results. 